\title{
ANÁLISE DAS TÉCNICAS DE FRACIONAMENTO DE AMOSTRAS BINÁRIAS DIFERENTES EM GRANULOMETRIA E DENSIDADE
}

\author{
I. S. STOPA ${ }^{1}$, F. C. NOGUEIRA ${ }^{2}$, C. A. PEREIRA ${ }^{3}$ \\ Universidade Federal de Ouro Preto \\ ORCID ID: https://orcid.org/ 0000-0002-2286-5465 ${ }^{1}$ \\ isabelastopa@hotmail.com ${ }^{1}$
}

Submetido 14/03/2020 - Aceito 06/06/2020

DOI: $10.15628 /$ holos.2020.9384

\section{RESUMO}

$\mathrm{Na}$ indústria mineral, a amostragem é um processo presente em todas as etapas do processo produtivo. Apesar disso, pouca importância é dada a ela, que é, muitas vezes, negligenciada. Nesse sentido, este trabalho foi desenvolvido com objetivo de avaliar as principais técnicas manuais de fracionamento de amostras: pilha cônica, pilha alongada, pazada fracionada, pazada alternada e quarteador Jones. Para tanto, utilizou-se seis misturas binárias sintéticas que variavam entre si em granulometria e densidade. Os resultados foram analisados por meio da distribuição mássica e do coeficiente de variação dos teores dos constituintes das amostras gêmeas geradas. De maneira geral, os métodos de fracionamento de amostras foram eficientes, uma vez que o valor médio das massas das amostras gêmeas geradas foi semelhante para todos os tipos de materiais testados. Além disso, os maiores coeficientes de variação dos teores puderam ser atribuídos, na maioria dos métodos testados, aos constituintes presentes em menor proporção da amostra $\left(C_{2}\right)$.

PALAVRAS-CHAVE: pilha cônica, pilha alongada, pazada alternada, pazada fracionada, quarteador Jones.

\section{ANALYSIS OF MANUAL FRACTIONATION TECHNIQUES FOR DIFFERENT BINARY SAMPLES IN GRANULOMETRY AND DENSITY}

\begin{abstract}
In the mineral industry, sampling is performed at all stages of the production process. Despite this, little importance is given to sampling, and it is often neglected. Considering this sense, this study was conducted to evaluate manual techniques relating to the fractionation of samples, namely, coning, long pile, alternate shovel, fractional shovel, and Jones riffle. Six synthetic binary mixtures, with varying grain sizes and densities, were used for this purpose. The results were analyzed via mass
\end{abstract} contents of the generated twin samples was calculated. The methods of fractionation of samples were efficient as the average values of the masses of the generated twin samples were similar for all types of materials tested. Additionally, the highest coefficients of variation of the contents in most of the tested methods could be attributed to the constituents present in smaller proportions to that of the sample $\left(C_{2}\right)$.

KEYWORDS: Coning, long pile, alternate shovel, fractional shovel, jones riffle. 


\section{INTRODUÇÃO}

A amostragem pode ser definida como uma sequência de operações para retirada de uma amostra ou de uma parte significativa de um todo sem que haja qualquer alteração em suas propriedades. Na indústria mineral, esse é um processo que está presente desde avaliação de depósitos minerais, passando pelo controle de qualidade das operações da mina e da usina e pela determinação dos parâmetros físico-químicos medidos nos laboratórios. Além disso, a amostragem possui fundamental importância para a garantia da qualidade dos produtos comercializados, o que significa dizer que uma amostragem mal conduzida pode resultar em grandes prejuízos financeiros para as empresas do setor. Apesar de sua importância, esse processo é muitas vezes negligenciado, o que se deve à falta de conhecimento da teoria e de investimentos em equipamentos e em treinamentos adequados (BORTOLETO, CHIEREGATI e OLIVEIRA, 2017).

Segundo Chaves (2012), é possível classificar a amostragem de acordo com o critério adotado para retirada das amostras, a saber: amostragem por incrementos e amostragem por fracionamento. No primeiro caso, a amostra final é formada pelo somatório dos incrementos (que são alíquotas representativas retiradas da população) a intervalos de tempo ou de percursos iguais ou não. Nesse caso, a amostra gerada é, então, homogeneizada e reduzida dando origem a amostra final. Já na amostragem por fracionamento, a população inicial é fracionada em duas ou mais partes iguais. Uma das amostras geradas é sucessivamente reduzida até a obtenção da amostra final.

Na prática, uma amostra dificilmente apresentará $100 \%$ das características do material do universo de onde foi retirada, devido ao erro fundamental da amostragem e aos outros erros decorrentes do processo em si (CHIEREGATI, 2007). O erro fundamental não pode ser eliminado, a menos que a amostra gerada seja o próprio universo. Nesse sentido, é necessária a elaboração de normas e critérios para amostragem e redução de amostras, adequando a cada situação uma solução que possibilite eliminar os erros ou, ao menos, minimizá-los (MAGRI e ORTIZ, 2000). Entretanto, admitindo-se um erro aceitável, é possível determinar uma massa mínima capaz de representar esse universo. A massa mínima é função do tipo de material, granulometria, teor do elemento de interesse e precisão desejada e baseia-se em ensaios preliminares e exploratórios, que permitem analisar a variabilidade do material (SAMPAIO e FRANÇA, 2007).

$\mathrm{Na}$ literatura existem descrições de diversas técnicas de fracionamento e redução de amostras. Tais métodos manuais podem ser realizados com material estático ou em movimento e, apesar de estarem associadas a muitos erros, devido aos baixos custos, estão fortemente presentes na indústria mineral, como a pilha cônica, pilha alongada, pazada fracionada, quarteador Jones e divisão por incrementos (SAMPAIO e FRANÇA, 2007). Esse fato motivou a realização deste trabalho, que teve como objetivo avaliar o desempenho das principais técnicas de amostragem manual, identificando o método mais adequado para redução de amostras que variavam entre si em densidade e granulometria. 


\section{METODOLOGIA}

Os experimentos foram realizados utilizando misturas binárias de quartzo colorido (amarelo, azul, branco, vermelho e roxo) e hematita. Para avaliar a influência da granulometria e da densidade das partículas no processo de homogeneização e quarteamento os testes foram agrupados em seis tipos distintos, conforme ilustrado na Tabela 1.

Para preparação do material, as amostras foram peneiradas a úmido e divididas em duas faixas granulométricas distintas denominadas neste trabalho como Faixa Fina $F(1,19-0,84 \mathrm{~mm}) \mathrm{e}$ Faixa Grossa G $(4,76-3,36 \mathrm{~mm})$. A proporção dos constituintes foi mantida fixa para todos os testes: $60 \%$ de quartzo cor $1\left(C_{1}\right)$ e $40 \%$ de quartzo cor 2 ou hematita $\left(C_{2}\right)$. Obedecendo a massa mínima representativa de cada mistura binária determinou-se para os testes do Tipo 1, Tipo 2, Tipo 3 e Tipo 4 a massa do lote igual a $1500 \mathrm{~g}$ e para os testes do Tipo 5 e Tipo 6 a massa do lote igual a $500 \mathrm{~g}$.

Tabela 1: Classificação das amostras quanto à granulometria, à densidade e à proporção dos constituintes

\begin{tabular}{|c|c|c|c|}
\hline AMOSTRA & GRANULOMETRIA & 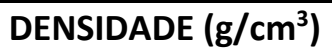 & MATERIAL \\
\hline TIPO 1 & Grosso - Grosso & $2,60-2,60$ & $\begin{array}{c}C_{1}=\text { Quartzo amarelo (60\%) } \\
C_{2}=\text { Quartzo azul }(40 \%)\end{array}$ \\
\hline TIPO 2 & Grosso - Grosso & $2,60-5,30$ & $\begin{array}{c}C_{1}=\text { Quartzo amarelo (60\%) } \\
C_{2}=\text { Hematita }(40 \%)\end{array}$ \\
\hline TIPO 3 & Fino - Grosso & $2,60-2,60$ & $\begin{array}{c}C_{1}=\text { Quartzo branco }(60 \%) \\
C_{2}=\text { Quartzo azul }(40 \%)\end{array}$ \\
\hline TIPO 4 & Fino - Grosso & $2,60-5,30$ & $\begin{array}{c}C_{1}=\text { Quartzo branco }(60 \%) \\
C_{2}=\text { Hematita }(40 \%)\end{array}$ \\
\hline TIPO 5 & Fino - Fino & $2,60-2,60$ & $\begin{array}{c}C_{1}=\text { Quartzo branco }(60 \%) \\
C_{2}=\text { Quartzo vermelho }(40 \%)\end{array}$ \\
\hline TIPO 6 & Fino - Fino & $2,60-5,30$ & $\begin{array}{c}\mathrm{C}_{1}=\text { Quartzo roxo }(60 \%) \\
\mathrm{C}_{2}=\text { Hematita }(40 \%)\end{array}$ \\
\hline
\end{tabular}

\subsection{Pilha Cônica}

Os testes de pilha cônica Teste 1, Teste 2 e Teste 3 foram realizados para os seis tipos de amostras, variando o número de ciclos de homogeneização em 5, 10 e 20, respectivamente.

Inicialmente, as duas partes constituintes de cada amostra foram dispostas separadamente sobre uma lona quadrada $(a=0,65 \mathrm{~m})$, configurando uma situação de segregação máxima. Homogeneizou-se o material levantando, alternadamente, os vértices da lona (ciclo), fazendo com que o material da base da pilha fosse disposto no topo. Após a formação de uma pilha cônica, o vértice do cone foi achatado e a pilha foi dividida em quatro partes iguais. As duas amostras gêmeas foram geradas tomando as partes diametralmente opostas. As etapas descritas estão ilustradas na Figura 1. 


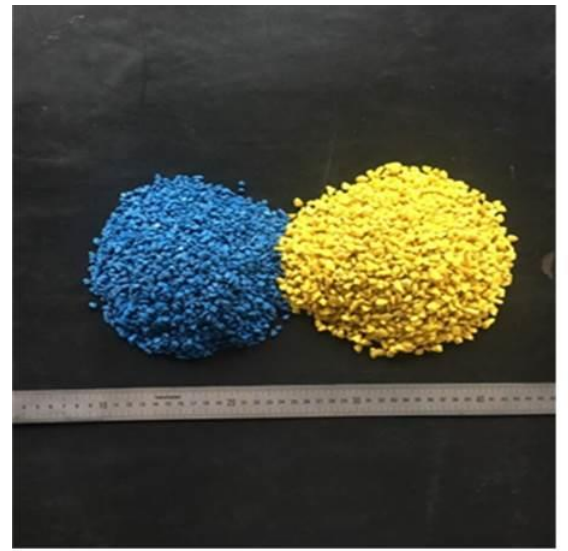

a)

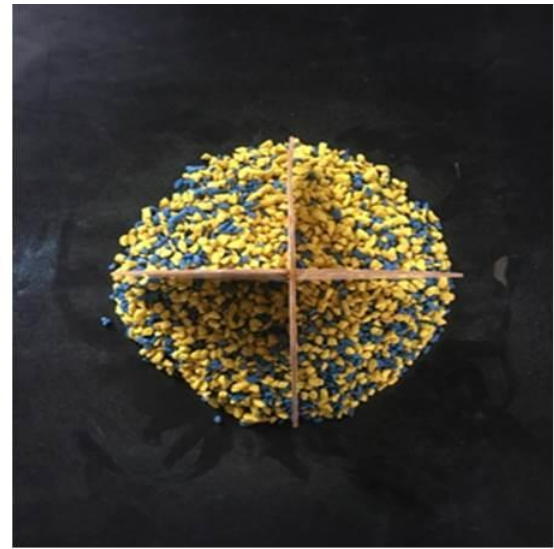

b)

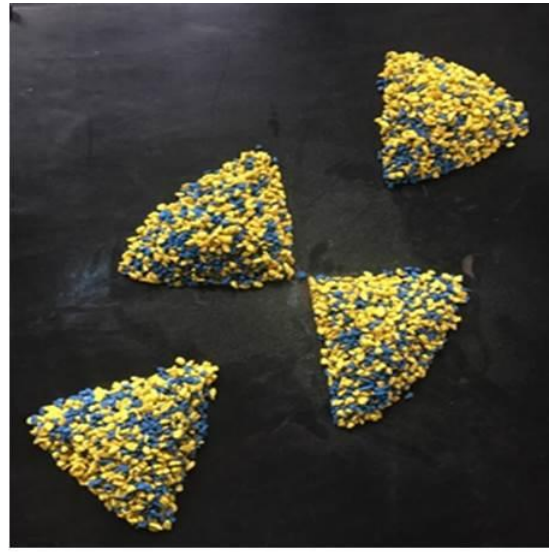

c)

Figura 1: Método da pilha cônica - a) segregação máxima; b) homogeneização; c) quarteamento

\subsection{Pilha Alongada}

Os testes de pilha alongada foram realizados para os seis tipos de amostras, variando o comprimento da pilha e mantendo fixo o número de incrementos que formaram cada uma das amostras gêmeas geradas.

A pilha alongada (Figura 2) foi formada dispondo as amostras ao longo do comprimento préestabelecido de $30 \mathrm{~cm}$ (Teste 1) ou $60 \mathrm{~cm}$ (Teste 2). A primeira fração do material foi distribuída da esquerda para direita, a segunda, da direita para esquerda e assim sucessivamente. A disposição do material foi feita de forma regular (velocidade constante), homogênea e a massa de cada fração retirada do lote inicial foi suficiente para descarregar ao longo do comprimento de toda a pilha

Construída a pilha, o material da extremidade foi retomado e redistribuído ao longo da mesma. Dividiu-se a pilha ao meio no sentido longitudinal e, posteriormente, em sete segmentos em sentido transversal de $8,6 \mathrm{~cm}$ para as pilhas de $60 \mathrm{~cm}$ e de $4,3 \mathrm{~cm}$ para as pilhas de $30 \mathrm{~cm}$.

$\mathrm{O}$ quarteamento foi realizado formando duas amostras, tomando-se para uma as alíquotas de índices ímpares e para a outra as com índices pares.

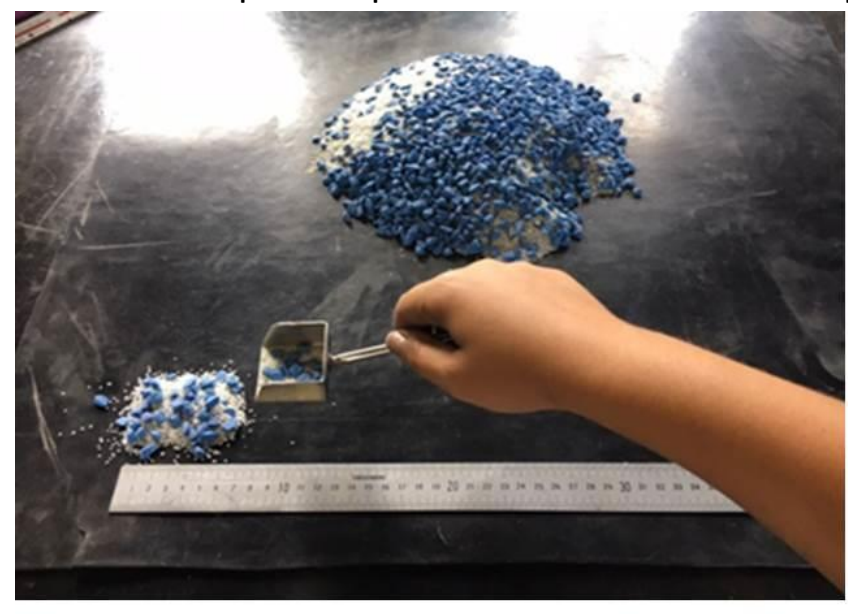

a)

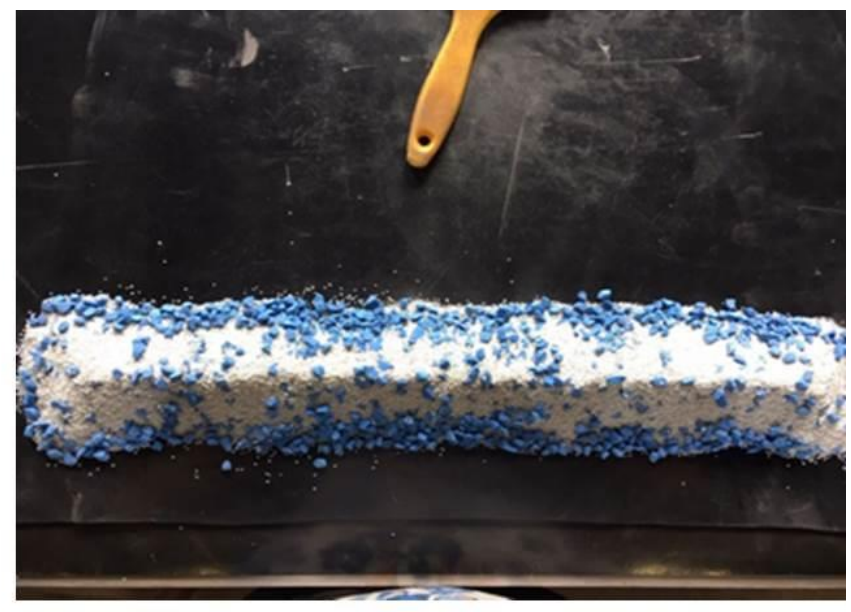

b)

Figura 2: Método da pilha alongada - a) formação da pilha alongada; b) pilha alongada amostra Tipo 3

HOLOS, Ano 36, v.6, e9384, 2020 


\subsection{Pazada Alternada}

Os testes de pazada alternada (Figura 3) foram realizados para os seis tipos de amostras. Formou-se uma pilha cônica no centro da lona e foram retiradas 30 alíquotas, seguindo o contorno da pilha e mantendo, em cada pazada, a massa média calculada pela Equação 1.

$M_{n}=\frac{M_{1}}{30 p}$

Onde:

$M_{n}$ :dimensão média da pazada;

$M_{l}$ : massa do lote;

$p$ : taxa de redução escolhida (número de pilhas a serem formadas).

Para os testes do Tipo 1, Tipo 2, Tipo 3 e Tipo $4\left(M_{l}=1500 \mathrm{~g}\right)$, a massa de enchimento média da pá foi igual a $M_{n}=25 \mathrm{~g}$. Já para os testes do Tipo 5 e Tipo $6\left(M_{l}=500 \mathrm{~g}\right)$, a massa de enchimento média da pá foi de $M_{n}=8,3 \mathrm{~g}$.

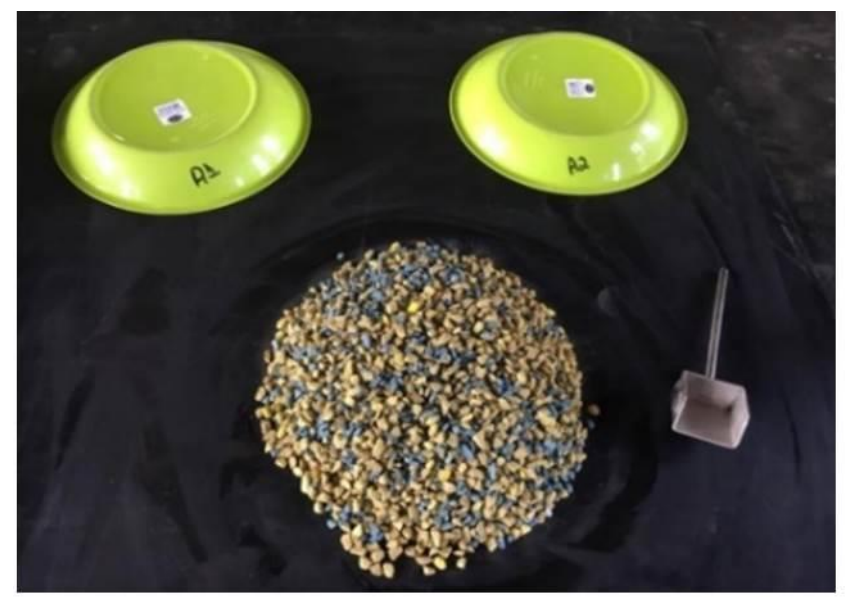

a)

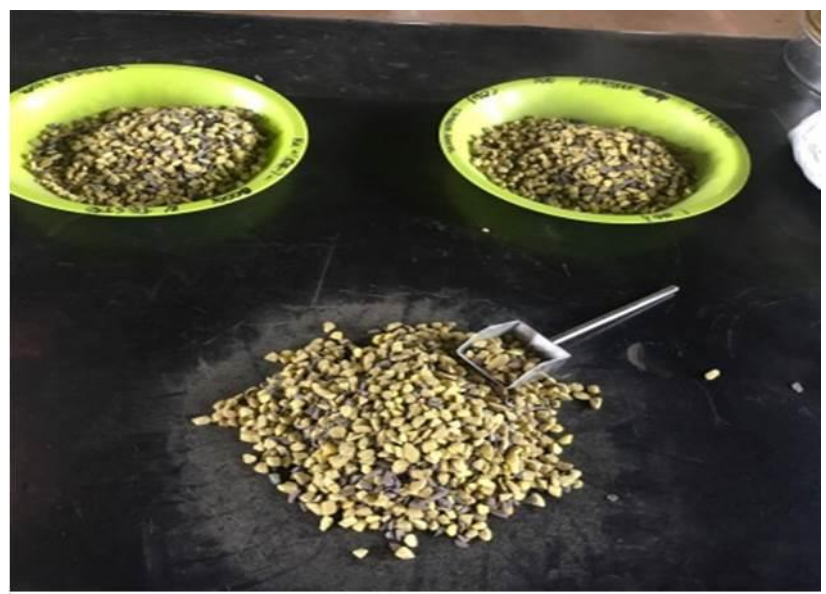

b)

Figura 3: Método da pazada alternada - a) pilha cônica; b) pazadas retiradas seguindo o contorno da pilha

\subsection{Pazada Fracionada}

Para os testes realizados utilizando o método de pazada fracionada foram geradas quatro amostras gêmeas ( $p=4)$, conforme apresentado na Figura 4. Para cada teste formou-se uma pilha cônica no centro da lona e retiraram-se, seguindo o contorno da pilha, 30 alíquotas, mantendo, em cada pazada, a massa média calculada. Para os testes do Tipo 1, Tipo 2, Tipo 3 e Tipo 4 ( $M_{l}=$ $1500 \mathrm{~g}$ ), a massa de enchimento média da pá foi igual a $M_{n}=12,4 \mathrm{~g}$. Já para os testes do Tipo $5 \mathrm{e}$ do Tipo $6 M_{l}=500 \mathrm{~g}$, a massa de enchimento média da pá foi $M_{n}=4,2 \mathrm{~g}$. 


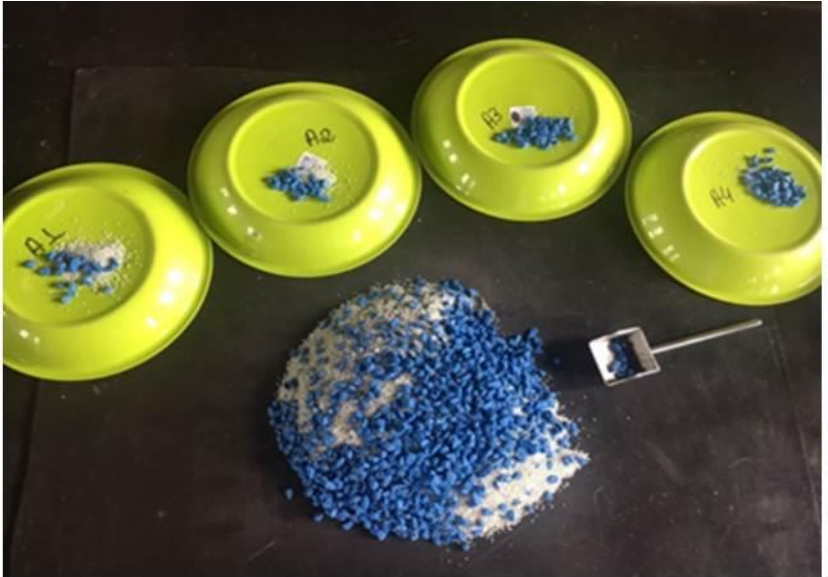

a)

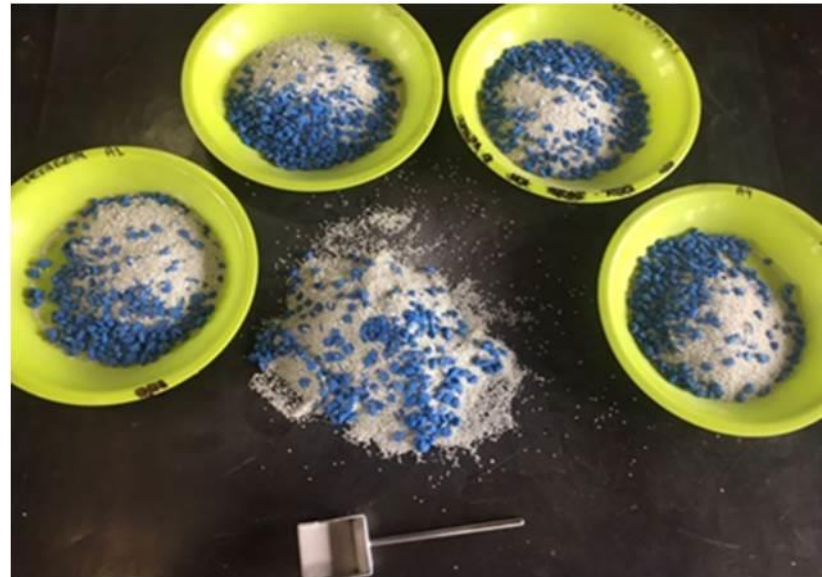

b)

Figura 4: Método da pazada fracionada Legenda - a) pilha cônica formada no centro da lona; b) quatro amostras gêmeas formadas

\subsection{Quarteador Jones}

Os testes utilizando o quarteador Jones foram realizados para os seis tipos de amostras, variando o número de calhas do equipamento em oito aberturas de $2,4 \mathrm{~cm}$ (Teste 1) ou em 16 aberturas de 1,2 cm (Teste 2) e, portanto, o número de incrementos que formaram cada uma das duas amostras.

Para cada teste, a amostra binária foi previamente homogeneizada, distribuída de forma contínua em um dos três compartimentos do Jones e disposta na posição central da grade do equipamento, conforme ilustrado Figura 6.

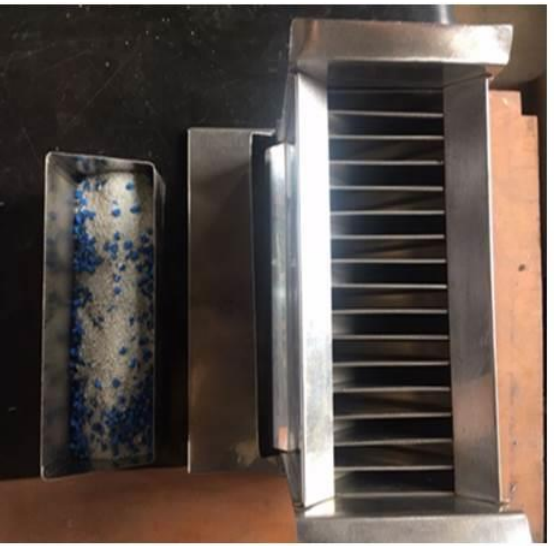

a)

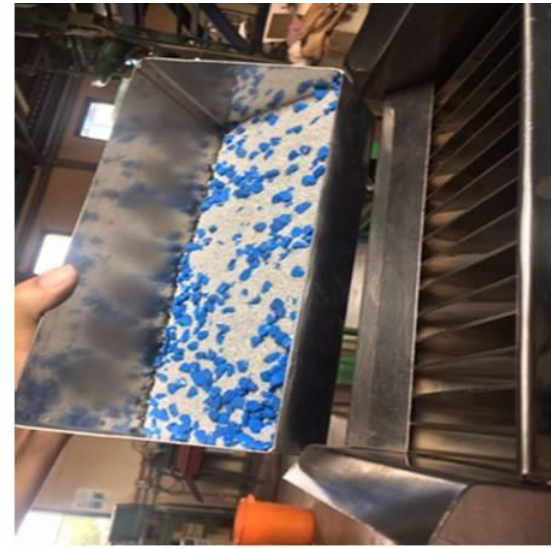

b)

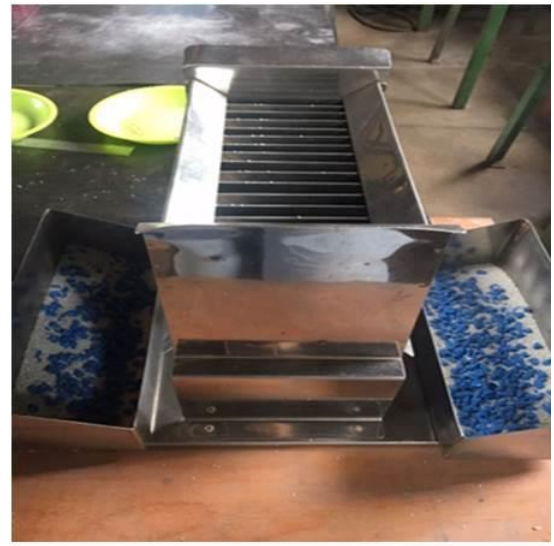

c)

Figura 6: Método de divisão no quarteador Jones - a) disposição do material em um dos compartimentos do Jones; b) quarteamento; c) amostras gêmeas formadas 


\subsection{Análise das Amostras Geradas}

O teor dos constituintes foi determinado a partir da análise e processamento de imagens no software Irfanview ${ }^{\circledR}$, que determinou a porcentagem de cada um dos dois constituintes presentes nas amostras, conforme ilustrado na Figura 7. Para cada análise, foram utilizadas 5 imagens de diferentes pontos da amostra e o teor de cada constituinte foi determinado pela média dos cinco valores gerados pelo programa.

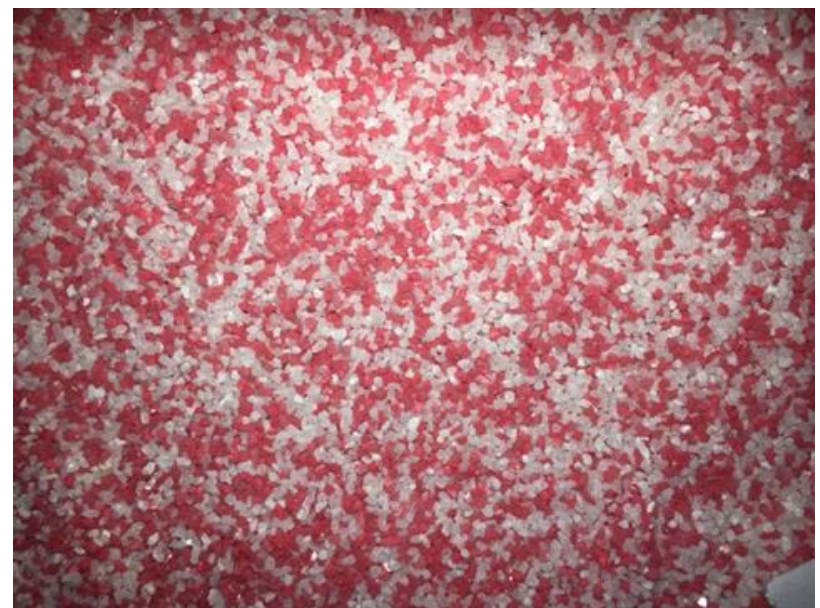

Figura 73 - Foto utilizada para análise de teor software Irfanview ${ }^{\circledR}$

Para análise da distribuição mássica dos testes realizados calculou-se o desvio padrão entre as massas normalizadas ( $\left(\mathrm{m}^{*}\right)$ ) e o coeficiente de variação (CV) dos teores dos constituintes.

\section{RESULTADOS E DISCUSSÃO}

As análises foram feitas com auxílio do software MINITAB $^{\circledR} 17$, com confiabilidade de $95 \%$, considerando a distribuição normal dos dados. Quanto maiores os valores tanto do desvio padrão das massas das amostras geradas quanto do coeficiente de variação de teor dos constituintes, mais distintas foram as amostras gêmeas e, consequentemente, menos eficiente o método naquela condição.

\subsection{Pilha Cônica}

Os resultados obtidos nos ensaios realizados por meio do método de pilha cônica estão expostos na Figura 8. 


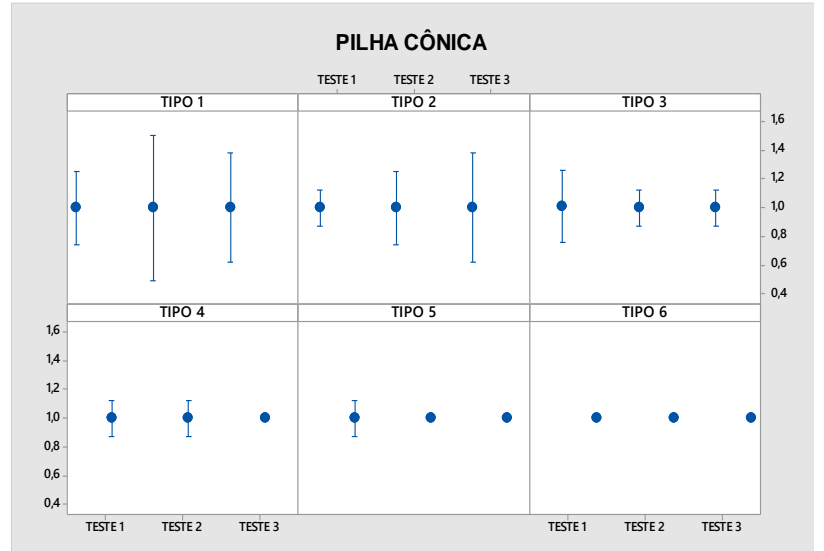

a)

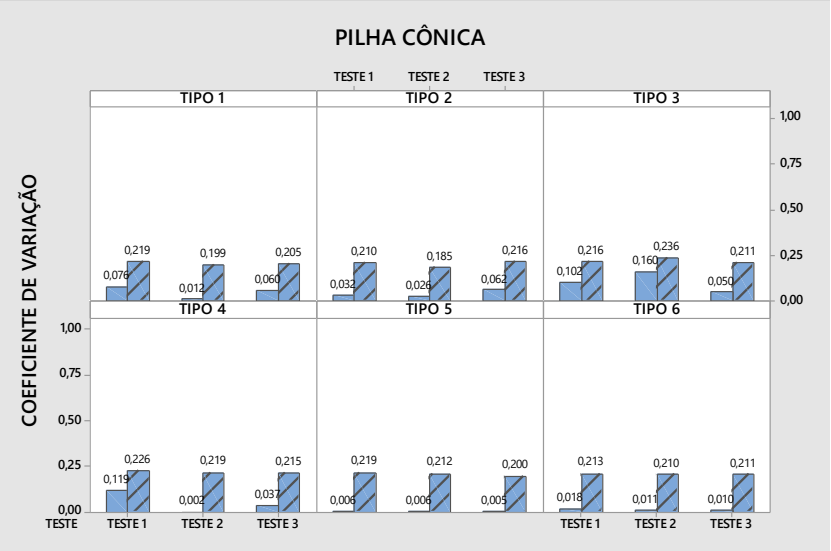

b)

Figura 8: Resultados pilha cônica a) gráfico de intervalos das massas normalizadas; b) coeficiente de variação (CV) de teor dos constituintes $C_{1}$ e $C_{2}$ nas amostras gêmeas

Analisando os dados apresentados no gráfico de intervalos da Figura 8a, foi possível observar que o valor médio das massas das amostras gêmeas geradas foi semelhante para todos os tipos de materiais testados. A dispersão dos dados dos ensaios realizados com o material do TIPO 5 e do TIPO 6, ambos constituídas por material do Tipo Fino-Fino, foram baixos. Já os intervalos de confiança das médias das massas dos experimentos realizados com os materiais do Tipo GrossoGrosso e Grosso-Fino foram, de maneira geral, os maiores. Isso pode ser atribuído à dificuldade de fracionamento das amostras constituídas por materiais grossos ou heterogêneos utilizando a cruzeta, o que resultou em uma distribuição menos uniforme entre as massas das amostras gêmeas geradas.

Em relação aos ciclos de homogeneização, os intervalos de confiança dos testes 1, 2 e 3 se sobrepuseram, isso indica que provavelmente a diferença do número de ciclos não foi significativa na distribuição mássica das amostras geradas.

Analisando os dados relacionados aos teores apresentados na Figura 8b, foi possível observar que os maiores coeficientes de variação puderam ser atribuídos aos constituintes presentes em menor proporção da amostra $\left(C_{2}\right)$.

Também foi possível observar, durante a realização dos testes, que os materiais do TIPO 3 e do TIPO 4 (Fino-Grosso) apresentaram o fenômeno de segregação (Figura 9) oriundo da heterogeneidade distribucional dos componentes da amostra. 


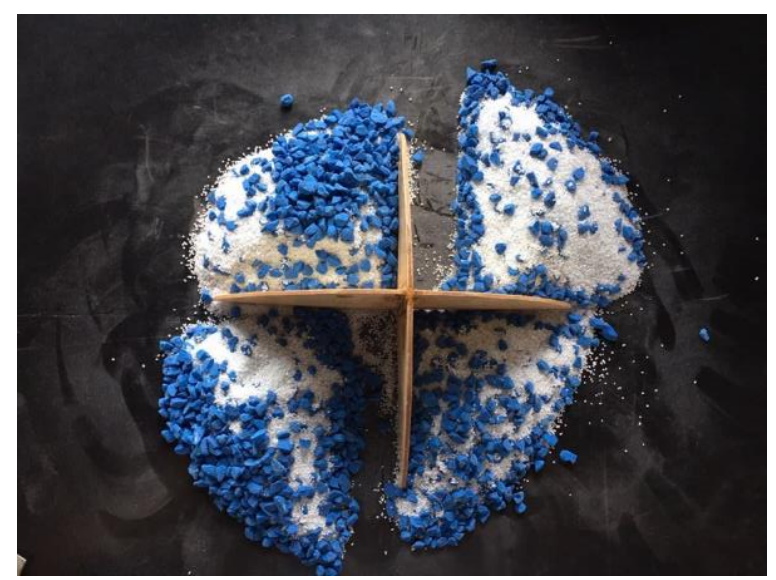

Figura 9: Efeito da segregação formado na pilha cônica

\subsection{Pilha Alongada}

Os resultados obtidos nos ensaios realizados por meio do método de pilha alongada estão expostos na Figura 10.

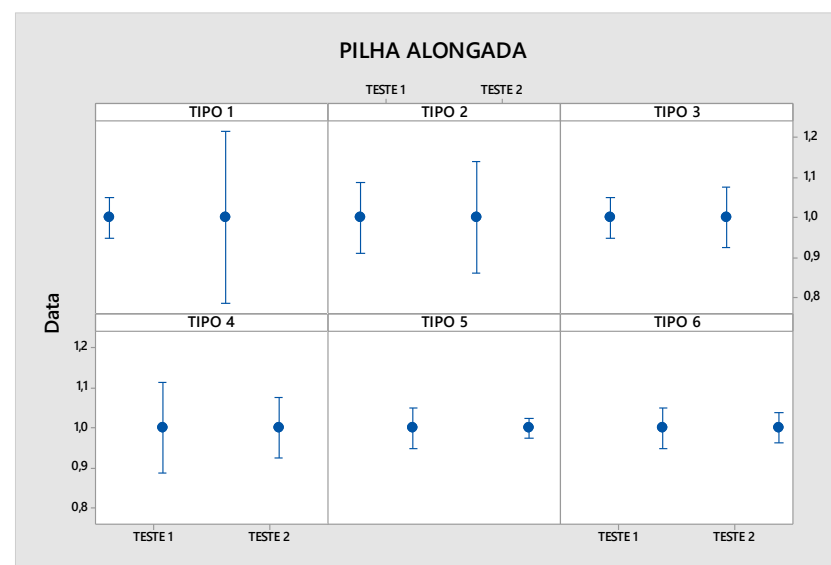

a)

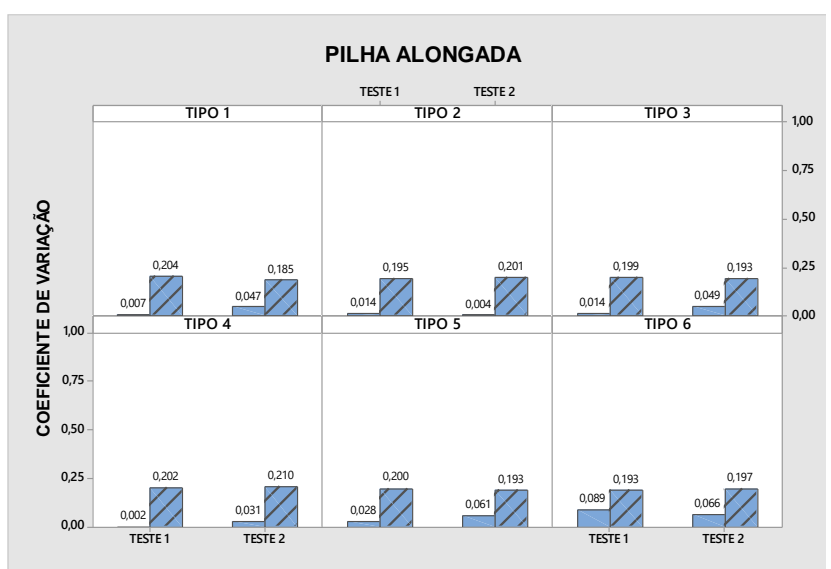

b)

Figura 10: Resultados pilha alongada a) gráfico de intervalos das massas normalizadas; $b$ ) coeficiente de variação (CV) de teor dos constituintes $C_{1}$ e $C_{2}$ das amostras gêmeas

Analisando os dados da Figura 10a, foi possível observar que a média das massas das amostras geradas foi semelhante para todos os tipos de materiais testados. A dispersão dos dados dos ensaios realizados com o material dos tipos Grosso-Grosso e Grosso-Fino foram, de maneira geral, os maiores, o que pôde ser atribuído à dificuldade em distribuir o material com granulometria grossa de forma regular durante todo comprimento da pilha alongada. Por outro lado, os experimentos realizados com o material do TIPO 5 e 6 (Fino-Fino) formavam uma pilha mais regular e os valores de massa das amostras geradas apresentaram menor dispersão.

Em relação ao tamanho das pilhas alongadas, os intervalos de confiança dos testes 1 e 2 se sobrepuseram, indicando que provavelmente a diferença do comprimento da pilha não foi significativa na distribuição mássica das amostras geradas. 
Analisando os dados apresentados no gráfico da Figura 10b relacionados aos teores, foi possível observar que os maiores coeficientes de variação puderam ser atribuídos aos constituintes presentes em menor proporção da amostra $\left(C_{2}\right)$.

\subsection{Pazada alternada e pazada fracionada}

Os resultados obtidos nos ensaios realizados por meio do método de pazada alternada e pazada fracionada estão expostos nos gráficos das Figuras 11 e 12, respectivamente.

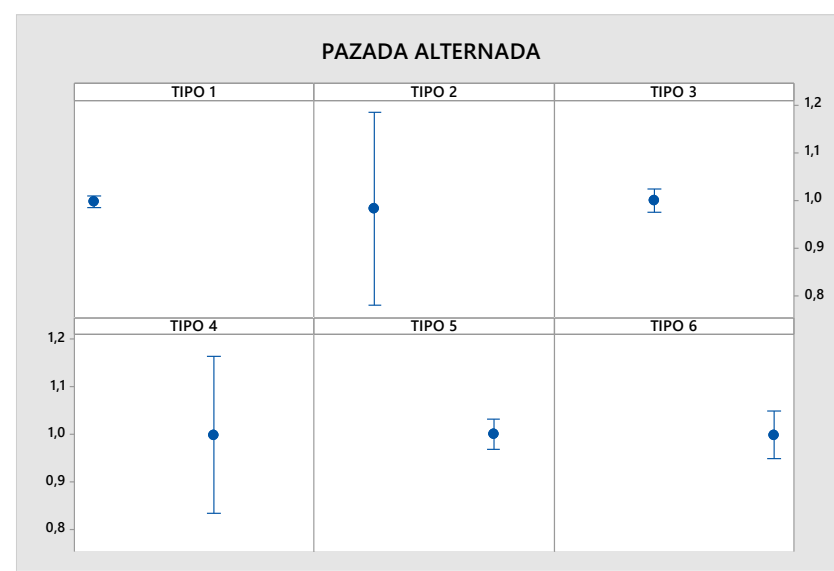

a)

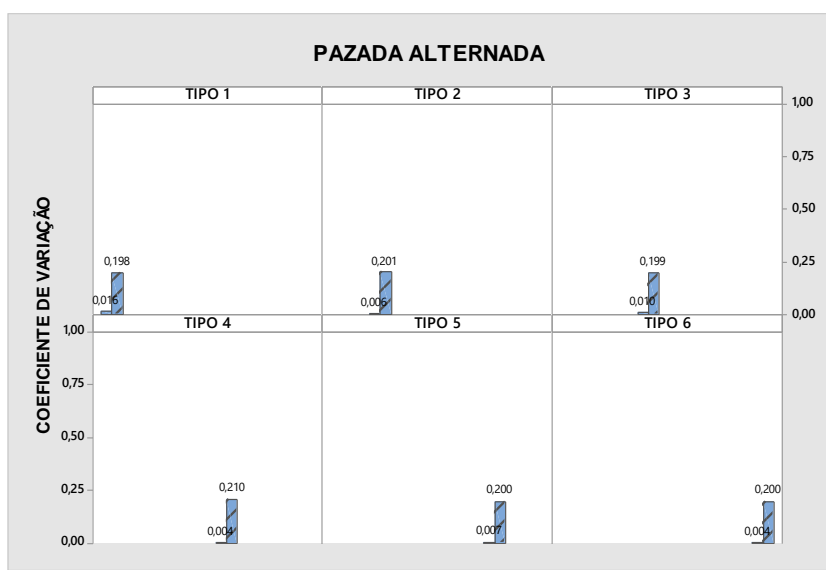

b)

Figura 11: Resultados pazada alternada a) gráfico de intervalos das massas normalizadas; b) coeficiente de variação (CV) de teor dos constituintes $C_{1}$ e $C_{2}$ das amostras gêmeas

Analisando os resultados obtidos pela pazada alternada (Figura 11a), foi possível observar que a média das massas das amostras geradas foi semelhante para todos os tipos de materiais testados. Também foi possível observar que a diferença de densidade dos constituintes foi responsável pelos maiores intervalos de confiança das médias das massas geradas. Isso pode ser explicado pelo fato de que as pazadas não uniformes com maiores frações de hematita $\left(C_{2}\right)$ pesavam mais, o que ocasionou tal variação na distribuição mássica.

Em relação aos teores (Figura 11b), observou-se que os maiores coeficientes de variação puderam ser atribuídos aos constituintes presentes em menor proporção da amostra $\left(C_{2}\right)$. 


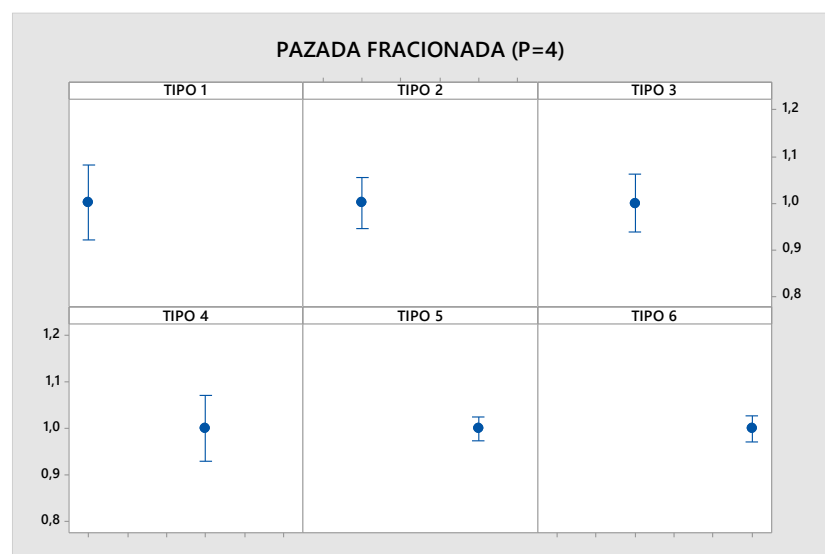

a)

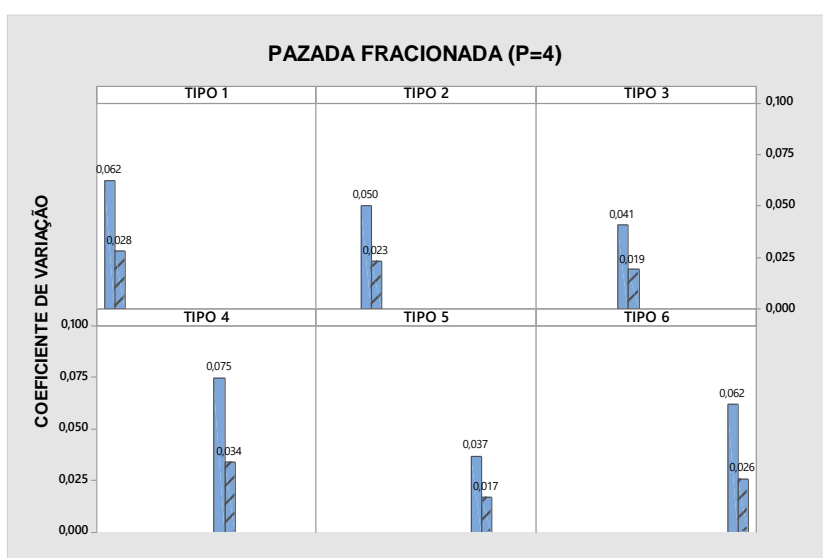

b)

Figura 12: Resultados pazada fracionada a) gráfico de intervalos para as massas normalizadas; b) coeficiente de variação (CV) de teor dos constituintes $C_{1}$ e $C_{2}$ das amostras gêmeas.

Também para pazada alternada (Figura 12a), foi possível observar que a média das massas das amostras geradas foi semelhante para todos os tipos de materiais testados. Os menores valores de dispersão dos dados foram obtidos para os experimentos realizados com o material do TIPO 5 e do TIPO 6, ambos compostos por Fino-Fino. Já o intervalo de confiança das médias das massas dos experimentos realizados com os outros tipos de materiais (Grosso-Grosso e Grosso-Fino) foram maiores. Isso pode ser explicado pela dificuldade em retirar as quatro pazadas de maneira uniforme, uma vez que as pilhas das quais foram retiradas as pazadas apresentavam segregação e heterogeneidade, logo as pazadas que possuíam maior quantidade de materiais grossos e mais densos pesavam mais.

Ao comparar os valores dos intervalos de confiança entre os experimentos realizados com a pazada alternada e com a pazada fracionada utilizando o mesmo material, foi possível observar que a distribuição mássica foi mais uniforme para a pazada alternada, evidenciando que manualmente é mais difícil formar um número maior de amostras com massa semelhante.

\subsection{Quarteador Jones}

Os resultados obtidos nos ensaios realizados por meio do método quarteador Jones estão expostos na Figura 13. 


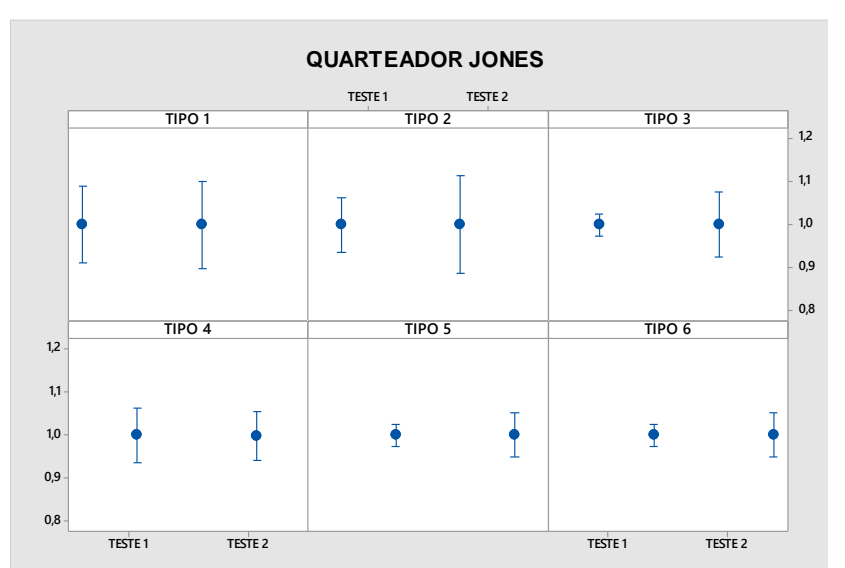

a)

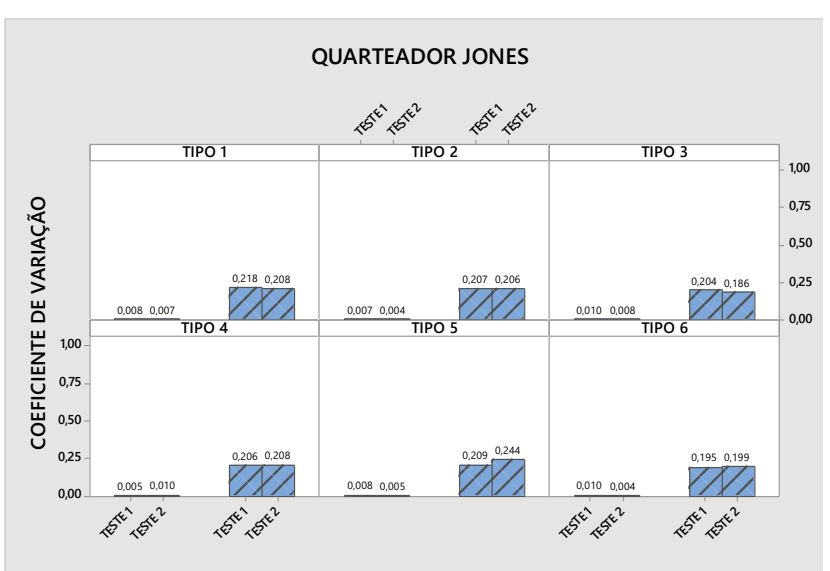

b)

Figura 13: Resultados quarteador Jones a) gráfico de intervalos para as massas normalizadas; b) coeficiente de variação (CV) de teor dos constituintes $C_{1}$ e $C_{2}$ das amostras gêmeas geradas

Analisando os resultados obtidos com o quarteador Jones (Figura 13a), foi possível observar que a média das massas das amostras geradas foi semelhante para todos os tipos de materiais testados.

Em relação ao número de calhas do Jones, os intervalos de confiança dos testes 1 e 2 se sobrepuseram, indicando que provavelmente a diferença do número de aberturas do equipamento não interferiu de forma significativa na média das massas das amostras geradas.

Em relação à granulometria do material, observou-se que os valores das médias das massas dos testes realizados com os materiais do TIPO 5 e 6 (ambos compostos por material Fino-Fino) foram menos dispersos. A distribuição dos outros materiais foi mais heterogênea uma vez que qualquer by-pass, por menor que fosse em quantidade, gerava uma distribuição não uniforme entre as massas das amostras gêmeas geradas.

Conforme apresentado pela Figura 13b, observou-se que os maiores coeficientes de variação dos teores puderam ser atribuídos aos constituintes presentes em menor proporção da amostra $\left(C_{2}\right)$. Ainda sobre o teor das amostras geradas, foi possível perceber que independente do número de calhas do equipamento (Teste 1 e Teste 2) o coeficiente de variação dos teores de cada constituinte $\mathrm{C}_{1}$ e $\mathrm{C}_{2}$ manteve-se semelhante.

\section{CONCLUSÃO}

Com base nos dados obtidos, pode-se concluir que para os métodos testados, com exceção da pazada fracionada, os maiores coeficientes de variação dos teores puderam ser atribuídos aos constituintes presentes em menor proporção da amostra $\left(C_{2}\right)$.

Em relação à distribuição mássica das amostras geradas, observou-se que para todos os métodos testados e para todos os materiais utilizados, a média das massas das amostras geradas foi semelhante. 
O fracionamento por pilha cônica foi eficiente para a divisão de materiais homogêneos e constituídos por partículas finas, apresentando os menores valores de dispersão entre os valores médios das massas normalizadas para os experimentos realizados com esse tipo de material.

Em relação à pilha alongada, a dispersão dos dados dos ensaios realizados com o material homogêneo e constituído por partículas finas foi baixa e a diferença do comprimento da pilha não influenciou de forma significativa nos valores médios das massas geradas.

Em relação ao método da pazada alternada e fracionada, as maiores dificuldades estão relacionadas à retirada de pazadas uniformes de pilhas constituídas por material heterogêneo. Comparando esses dois métodos, foi possível observar que a pazada alternada apresentou menor dispersão dos valores das médias das massas geradas, evidenciando que manualmente é mais difícil formar um número maior de amostras com massa semelhante.

Para o quarteador Jones, observou-se que a diferença do número de aberturas do equipamento não interferiu de forma significativa na média das massas das amostras geradas para os materiais testados.

\section{REFERÊNCIAS}

BORTOLETO, D. A; CHIEREGATI A. C; OLIVEIRA R. C. Otimização de protocolos de amostragem para minério de alumínio. Revista Holos, Ano 33. Vol 6, 2017.

CHIEREGATI, A. C. Reconciliação pró-ativa em empreendimentos mineiros. REM: Revista Escola de Minas, Ouro Preto, v.61, n.3, p. 297-302. 2007.

CHAVES, A. P. Manuseio de sólidos granulados. 2a edição, Editora Oficina de textos, 2012.

MAGRI, E. V.; ORTIZ, J. C. Estimation of economic losses due to poor blast hole sampling in open pits, In: Proceedings of Geostat 2000 (CD-ROM), 2000. p. 1-10.

SAMPAIO J. A.; FRANÇA, S. A. Tratamento de minérios: Práticas laboratoriais. CETEM/MCT, 2007.

\section{COMO CITAR ESTE ARTIGO:}

Stopa, I. S., Nogueira, F. C., Pereira, C. A. (2020). Análise das técnicas de fracionamento de amostras binárias diferentes em granulometria e densidade. Holos. 36(6), 1-14.

\section{SOBRE OS AUTORES}

\section{S. STOPA}

Mestre em Engenharia Mineral pelo programa de pós-graduação de Engenharia Mineral da Universidade Federal de Ouro Preto. E-mail: isabelastopa@hotmail.com

ORCID ID: https://orcid.org/0000-0002-2286-5465 


\section{F. C. NOGUEIRA}

Doutora em Engenharia Minaral na Universidade Federal de Ouro Preto - Departameto de Engenharia de Minas - Ouro Preto - MG. E-mail: franciellenoguera@yahoo.com.br

ORCID ID: http://orcid.org/0000-0001-5912-011X

\section{A. PEREIRA}

Professor Doutor em Engenharia Minaral na Universidade Federal de Ouro Preto - Departameto de Engenharia de Minas - Ouro Preto - MG. E-mail: pereiraufop@gmail.com

ORCID ID: http://orcid.org/0000-0003-0510-0257

Editor(a) Responsável: Francinaide de Lima Silva Nascimento

Pareceristas Ad Hoc: MAURO MEYER E FÁBIO DE SÃO JOSÉ

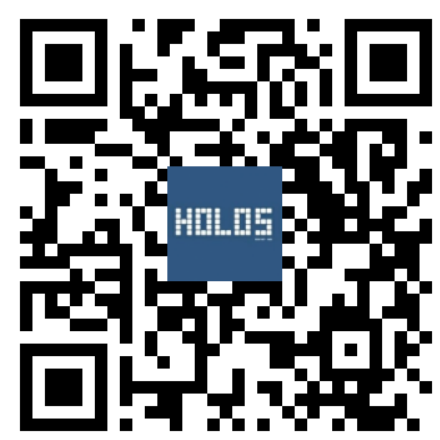

